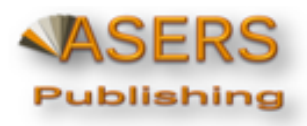

DOI: https://doi.org/10.14505/jmef.v4.2(7).0X

\title{
Insurance-markets Equilibrium with Sequential Non-convex Straight-time and Over-time Labor Supply
}

\author{
Aleksandar Vasilev \\ Independent Researcher \\ alvasilev@yahoo.com
}

\begin{abstract}
This note describes the lottery - and insurance-market equilibrium in an economy with non-convex straight-time and overtime employment. In contrast to Hansen and Sargent (1988), the overtime-decision is a sequential one. This requires two separate insurance market to operate, one for straight-time work, and one for overtime. In addition, given that the labor choice for regular and overtime hours is made in succession, the insurance market for overtime needs to open once the insurance market has closed. This segmentation and sequentiality of insurance markets operation is a new result in the literature and a direct consequence of the sequential nature of the overtime labor decision.
\end{abstract}

Keywords: Indivisible labor, straight time, overtime, sequential lotteries, insurance.

JEL Classification Codes: E1, J2, J4.

\section{Introduction and Motivation}

Changes in hours account for approximately two-thirds of the cyclical output volatility in the standard real business cycle model (Cooley and Prescott (1995), Kydland (1995)). Those hours, however, are assumed to be supplied as normal hours (straight-time), while overtime has been largely ignored. This comes as stark contrast to data, where overtime is widely featured. After all, overtime carries a significant wage premium (Hansen and Sargent (1988)). Employers in sectors using semi-skilled workers might still prefer 
to pay a higher wage to a worker who is already familiar with the tasks, instead of incurring the costs of searching for a new hire, and having to explain the particulars of the job to that person. This paper adds to the literature by taking seriously the problem of non-convex labor supply decision in an economy with both straight time and overtime. In contrast to Hansen and Sargent (1988), the paper models this as both a non-convex, and a sequential decision. The paper will try to uncover whether this two-stage non-convex labor supply decision, and the sequential (non-convex) overtime labor decision margin in particular, could provide new implications for business cycle fluctuations.

In an earlier paper, Vasilev (2016) extends Hansen and Sargent (1988) with a sequential overtime decision. More specifically, the problem is one of two-stage non-convex labor supply decisions in an economy where agents first decide whether to participate in the labor market or stay unemployed, and then, conditional on being hired, need to decide whether they will work only the fulltime equivalent, or engage in overtime hours. Vasilev (2016) then aggregates over individual households utility functions, and finds that the resulting utility representation features interesting nonlinearities that were not present at individual level. The aggregate representation features dis-utilities of both regular and overtime hours that are dependent on the other types of hours. Therefore, instead of changing from one to infinity, as in Hansen and Sargent (1988), with a sequential non-convexity, the elasticity of labor supply for overtime work is a function of overall participation rate, and the elasticity of labor supply for full-time work depends on the share of workers doing overtime. The aggregate utility function derived in Vasilev (2016) allows for a different transmission mechanism of shocks as compared to the one in Hansen and Sargent (1988).

In contrast to this earlier study, the focus of the present note falls 
on the lottery- and insurance-market equilibrium for the setup in Vasilev (2016). In the presence of non-convex labor supply for straight-time, and when the overtime labor decision is assumed to be sequential, the setup requires two separate insurance market to operate, one for straight-time work, and one for overtime. In addition, given that the labor choice for regular and overtime hours is made in succession, the insurance market for overtime needs to open only after the insurance market for straight-time has closed. This sequentiality of insurance markets operation is a new result in the literature and a direct consequence of the sequential nature of the overtime labor decision.

\section{Model Setup}

The basis of the model is the setup described in Vasilev (2016), but extended to incorporate an institution selling unemployment insurance. The economy is static, there is no physical capital, and agents face a sequential discrete labor supply decision. There is a large number of identical one-member households, indexed by $i$ and distributed uniformly on the $[0,1]$ interval. In the exposition below, we will use small case letters to denote individual variables and suppress the index $i$ to save on notation.

\subsection{Household's problem}

Each one-member household maximizes the following utility function:

$$
U(c, l)=\ln c+\alpha \ln l,
$$

where $c$ denotes consumption of market output, $l$ is the leisure enjoyed by each individual household, and $\alpha>0$ is the relative weight attached to utility of leisure. Each household is endowed 
with a time endowment of unity..$^{1}$ Each household faces a sequential labor-supply decision. In stage 1, each household must decide whether to work or not. In stage 2 , conditional on working, the household decides whether to work straight-time $(\bar{h})$, or overtime $\left(h^{o}\right) t^{2}$ The wage rate is $w$ for straight-time hours and $w^{o}$ for overtime hours, with $w^{o}>w$. Finally, the households own the firm in the market economy, and are entitled an equal share of the profit $(\pi)$, where $\int_{0}^{1} \pi d i=\Pi$, hence $\pi=\Pi !^{3}$

Household's utility maximization problem of choosing $\{c, h\}$ optimally by taking $\left\{w, w^{o}, \pi\right\}$ as given, can be split into three subcases: $c^{u}$ will denote consumption of households that do not work, with $c^{u}=\pi$ and $l^{u}=1$. Similarly, full-time workers $4^{4}$ enjoy

$$
c^{e, f}=w \bar{h}+\pi
$$

and

$$
l^{e, f}=1-\bar{h},
$$

and overtime workers enjoy

$$
c^{e, o}=w \bar{h}+w^{o} h^{o}+\pi
$$

and

$$
l^{e, o}=1-\bar{h}-h^{o}
$$

(where superscript $e$ denotes workers, $f$ refers to the full-time workers, and $o$ denotes overtime workers).

\footnotetext{
${ }^{1}$ The separability of consumption and leisure is not a crucial assumption for the results that follow. A more general, non-separable, utility representation, does not generate new results, while significantly complicates the algebraic derivations, and thus interferes with model tractability.

${ }^{2}$ Those are taken as given, e.g. $\bar{h}=40$ hours per week, and $h^{\circ}=8$ hours of overtime work.

${ }^{3}$ This is more of a technical assumption, which is imposed to guarantee that even if a household does not supply any labor, it will enjoy a positive consumption.

${ }^{4}$ In what follows, "full-time" and "straight-time" will be used interchangeably.
} 


\subsection{Firms}

There is a representative firm producing a homogeneous final consumption good (its price is normalized to unity). The production function is given by

$$
Y=F\left(\bar{H}, H^{o}\right), F_{1}>0, F_{2}>0, F_{11}<0, F_{22}<0, F_{12}=0 .
$$

There are two capacity constraint:

(i) If all households work straight-time only, the marginal product of a regular hour of work is zero, i.e. $F_{1}(\bar{h})=0$;

(ii) If every employee works overtime, the marginal productivity of overtime labor also becomes equal to zero, i.e. $F_{2}\left(h^{o}\right)=0.5$

As in Hansen and Sargent (1988), the firm treats straight-time labor and overtime labor differently. 6

The firm acts competitively by taking wages $\left\{w, w^{o}\right\}$ as given, and chooses hours $\{\bar{H}\}$ and $\left\{H^{\circ}\right\}$ to maximize profit:

$$
\max _{\bar{H}, H^{o}} F\left(\bar{H}, H^{o}\right)-w \bar{H}-w^{o} H^{o} \quad \text { s.t. } \quad \bar{H} \geq 0, H^{o} \geq 0 .
$$

Vasilev (2016) establishes that in equilibrium, $q$ share of the onemember households will decide to work, and in the second stage $\lambda$ share of those who decided to work in the previous stage, or $q \lambda$ of all the households, will decide to work overtime.

\section{Insurance Markets}

An alternative way to represent the labor selection arrangement in the market sector is to regard workers as participants in

\footnotetext{
${ }^{5}$ This is a technical assumption to guarantee that in equilibrium there will be positive economic profits.

${ }^{6}$ This is due to the presence of some labor frictions, which are not explicitly modelled here. To simplify the analysis we assume the two types of hours are not substitutes.
} 
a lottery with the proportion employed equal to the probability of being selected for work. Therefore, we can introduce insurance markets, and allow households to buy insurance, which would allow them to equalize the actual income received independent of the employment status. iven the observed difference in the wages for straight-time and overtime hours, and the sequential nature of overtime labor supply decision, sequential and segmented insurance markets are also needed in order to provide actuarially fair insurance.

More specifically, the structure of the insurance industry is as follows: There is one representative insurance company for straighttime hours, and one for overtime The two companies are segmented and operate in sequence. At the beginning of each period, the households decide if and how much insurance to buy against the probability of being chosen for straight-time work. Then, the company closes, and the insurance company for overtime work opens. In both cases insurance costs $p^{j}$ per unit, $j=q, \lambda$, and provides one unit of income if the household is not working. We can think of insurance as bonds that pay out only in case the household is not chosen for work. Thus, household will also choose the quantity of insurance to purchase $b^{j}$. This setup requires that the overtime insurance company insures workers who have already been selected for work in the first stage. In this sense, the insurance markets are segmented as well.

Without the segmented and sequential nature of the insurance markets described above, insurance will not be actuarially fair, one of the groups will face better odds versus price, the company will not be able to break even, and/or at least one type of households will not be able to buy full insurance, which would completely smooth consumption across employment states, given the non-convexity constraint of labor supply. Furthermore, as pointed out in Hansen (1985), the plausibility of this insurance market seg- 
mentation result depends crucially on the fact that probabilities $q$ and $\lambda$ are perfectly observable to everyone, and that the contracts written are perfectly enforceable. Also, who has won and who has lost the lottery is assumed to be perfect knowledge. Lastly, everyone will always announce truthfully the same $q$ and $\lambda$ to each of the insurance companies.

\subsection{Insurance company for straight time}

The insurance company for straight time maximizes profit. The company services all households. It receives revenue if a household is working and makes payment if it is not. More specifically, the proportion of people working full-time contribute towards the unemployment benefits pool, which are then distributed of benefits to the unemployed. The amount of insurance sold is a solution to the following problem. Taking $p^{q}(i)$ as given, $b^{q}(i)$ solves

$$
\max _{b^{q}(i)} q(i) p^{q}(i) b^{q}(i)-[1-q(i)] b^{q}(i) .
$$

With free entry profits are zero, hence

$$
q(i) p^{q}(i) b^{q}(i)-[1-q(i)] b^{q}(i)=0,
$$

hence the insurance market for each household clears.

\subsection{Insurance company for overtime}

The insurance company for overtime also maximizes profit. The company opens once the insurance company has already closed, and services only the households that have been selected for straight time in the first stage. It receives revenue if a household is working overtime and makes payment if it is not. More specifically, the proportion of people working full-time contribute towards the unemployment benefits pool, which are then distributed to the 
workers who only work straight time. The amount of insurance sold is a solution to the following problem. Taking $p^{\lambda}(i)$ as given, $b^{\lambda}(i)$ solves

$$
\max _{b^{\lambda}(i)} \lambda(i) p^{\lambda}(i) b^{\lambda}(i)-[1-\lambda(i)] b^{\lambda}(i)
$$

With free entry profits are zero, hence

$$
\lambda(i) p^{\lambda}(i) b^{\lambda}(i)-[1-\lambda(i)] b^{\lambda}(i)=0 .
$$

This condition implicitly clears the insurance market for each household, conditional on being selected in the first stage for work.

In the next section, the equilibrium with lotteries and no insurance markets is presented and discussed first, and then the setup is extended to incorporate a regime with insurance.

\section{Definition of the DCE with lotteries}

\subsection{Decentralized Competitive Equilibrium (DCE) with lotteries}

A competitive Equilibrium with sequential Lotteries for this economy is a list

$$
\left(c^{u}(i), c^{e, f}(i), c^{e, o}(i), \lambda(i), q(i), \bar{h}, h^{o}, w, w^{o}, \pi\right)
$$

such that the following conditions are fulfilled.

1. Consumers maximization condition. Taking prices $w, w^{o}, \pi$ as given, for each $i$, the sequence

$$
\sigma=\left(c^{u}(i), c^{e, f}(i), c^{e, o}(i), \lambda(i), q(i), \bar{h}, h^{o}, w, w^{o}, \pi\right)
$$

solves the maximization problem

$$
\begin{gathered}
\max _{\sigma \in \Sigma} q(i)\left\{\lambda(i)\left[\ln \left(c^{e, f}\right)+\alpha \ln (1-\bar{h})\right]+(1-\lambda(i))\left[\ln \left(c^{e, o}\right)+\right.\right. \\
\left.\left.+\alpha \ln \left(1-\bar{h}-h^{o}\right)\right]\right\}+[1-q(i)] \ln \left(c^{u}\right)
\end{gathered}
$$


s.t

$$
\begin{array}{r}
q(i)\left[\lambda(i) c^{e, f}+(1-\lambda(i)) c^{e, o}(i)\right]+[1-q(i)] c^{u}= \\
=q(i) w \bar{h}+q(i)(1-\lambda(i)) w^{o} h^{o}+\pi
\end{array}
$$

with

$$
c^{e, f}(i), c^{e, o}, c^{u} \geq 0,0 \leq q(i), \lambda(i) \leq 1, \forall i,
$$

where $\Sigma$ is the constraint defined by relations (11)-(12) above.

2. Firm maximization condition. Taking prices $w, w^{o}, \pi$ as given,

$$
\max _{\bar{H}, H^{o}} F\left(\bar{H}, H^{o}\right)-w \bar{H}-w^{o} H^{o} \quad \text { s.t. } \quad \bar{H} \geq 0, H^{o} \geq 0 .(13)
$$

3. Market-clearing conditions. We have

$$
\begin{array}{r}
\int_{i} q(i) \bar{h} d i=\bar{H}, \\
\int_{i} q(i)[1-\lambda(i)] h^{o} d i=H^{o}, \\
\int_{i}\left\{q(i)\left[\lambda(i) c^{e, f}+(1-\lambda(i)) c^{e, o}\right]+\right. \\
\left.+[1-q(i)] c^{u}\right\} d i=F\left(\bar{H}, H^{o}\right),
\end{array}
$$

where the last equation describes clearing in the goods market.

\subsection{Characterizing the DCE}

The household's problem is as follows:

$$
\begin{gathered}
\mathcal{L}=\max _{\sigma \in \Sigma} q(i)\left\{\lambda(i)\left[\ln \left(c^{e, f}\right)+\alpha \ln (1-\bar{h})\right]+(1-\lambda(i)) *\right. \\
\left.*\left[\ln \left(c^{e, o}\right)+\alpha \ln \left(1-\bar{h}-h^{o}\right)\right]\right\}+[1-q(i)] \ln \left(c^{u}\right)- \\
-\mu\left[q(i)\left[\lambda(i) c^{e, f}+(1-\lambda(i)) c^{e, o}(i)\right]++[1-q(i)] c^{u}-\right. \\
\left.-q(i) w \bar{h}-q(i)(1-\lambda(i)) w^{o} h^{o}-\pi\right],
\end{gathered}
$$


where $\mu$ is the Lagrangian multiplier in front of the households' budget constraint. The first-order optimality conditions are as follows:

$$
\begin{array}{r}
c^{u}(i): \frac{1}{c^{u}(i)}=\mu, \forall i, \\
c^{e, f}(i): \frac{1}{c^{e, f}(i)}=\mu, \forall i, \\
c^{e, o}(i): \frac{1}{c^{e, o}(i)}=\mu, \forall i .
\end{array}
$$

It follows that

$$
c^{u}(i)=c^{e, f}(i)=c^{e, o}(i)=1 / \mu, \forall i .
$$

We simplify the Lagrangian by suppressing all consumption superscripts and $i$ notation in the derivations to follow

$$
\begin{gathered}
q(i): \lambda \alpha \ln (1-\bar{h})+(1-\lambda) \alpha \ln \left(1-\bar{h}-h^{o}\right)= \\
=-\mu\left[w \bar{h}+(1-\lambda) w^{o} h^{o}\right] \\
\lambda(i): q \alpha\left[\ln (1-\bar{h})-\ln \left(1-\bar{h}-h^{o}\right)\right]=\mu q w^{o} h^{o}
\end{gathered}
$$

or

$$
\frac{\ln (1-\bar{h})-\ln \left(1-\bar{h}-h^{o}\right)}{c h^{o}}=w^{o} .
$$

This equation is a discrete version of the marginal product of labor equals the marginal rate of substitution. It implicitly characterizes optimal $\lambda$.

Note that it is optimal from the benevolent planner/government point of view to choose randomly $q$ and $\lambda$ and to introduce uncertainty. With randomization, choice sets are convexified, and thus market completeness is achieved. A household is exposed to risk: first, it can be chosen to work with some probability; second, conditional on being chosen to work, it can be picked to provide 
overtime labor services. Given the risk in the economic environment, it would be optimal to have insurance. The government sells employment lotteries, and individuals will buy insurance to cover any risk exposure. With insurance, the employer pays wage to individuals only if they work. Now we extend the commodity space a little bit to include insurance markets explicitly.

5. Decentralized Competitive Equilibrium (DCE) with insurance markets

\subsection{Definition of the DCE with insurance markets}

A competitive Equilibrium with sequential Lotteries and insurance markets for this economy is a list

$$
\begin{gathered}
\left(c^{u}(i), c^{e, f}(i), c^{e, o}(i), \lambda(i), q(i), \bar{h}, h^{o},\right. \\
\left.w, w^{o}, \pi, b^{q}(i), b^{\lambda}(i), p^{q}(i), p^{\lambda}(i)\right)
\end{gathered}
$$

such that the following conditions are fulfilled.

1. Consumers maximization condition. Taking prices $w, w^{o}, \pi$ as given, for each $i$, the sequence

$$
\sigma=\left(c^{u}(i), c^{e, f}(i), c^{e, o}(i), \lambda(i), q(i), \bar{h}, h^{o}\right)
$$

solves the maximization problem

$$
\begin{array}{r}
\max _{\sigma \in \Sigma} q(i)\left\{\lambda(i)\left[\ln \left(c^{e, f}\right)+\alpha \ln (1-\bar{h})\right]+(1-\lambda(i)) *\right. \\
\left.*\left[\ln \left(c^{e, o}\right)+\alpha \ln \left(1-\bar{h}-h^{o}\right)\right]\right\}+[1-q(i)] \ln \left(c^{u}\right)
\end{array}
$$

s.t

$$
\begin{array}{r}
c^{e, f}+b^{q} p^{q}=b^{\lambda}+w \bar{h}+\pi \\
c^{e, o}+b^{q} p^{q}+b^{\lambda} p^{\lambda}=w \bar{h}+w^{o} h^{o}+\pi \\
c^{u}=b^{q}+\pi \\
c^{e, f}(i), c^{e, o}, c^{u} \geq 0,0 \leq q(i), \lambda(i) \leq 1, \forall i,
\end{array}
$$


where $\Sigma$ is the constraint defined by the above relations.

The interpretation of the constraints is as follows: In the first stage, workers buy unemployment insurance, while unemployed households will receive the payout (unemployment benefits, denoted by $\left.b^{q}\right)$. Then, conditional on being employed, overtime workers will buy non-overtime insurance (in case they are not chosen to work overtime), while full-time workers will receive the payout $b^{\lambda}$. Thus, overtime workers need to buy two types of insurance. Also, in equilibrium, it must be that $b^{q}=q w \bar{h}$, and $b^{\lambda}=(1-\lambda) w^{o} h^{o}$.

\subsection{Characterization of the DCE with insurance markets}

Before optimizing, simplify he constraint set by substituting out $b^{q}$ from the budget constraint in the state the household is unemployed to obtain

$$
b^{q}=c^{u}-\pi .
$$

Next, plug the obtained expression in the budget constraint in the state when the household is employed full-time to obtain

$$
c^{e, f}+p^{q}\left(c^{u}-\pi\right)=b^{\lambda}+w \bar{h}+\pi .
$$

Now substitute out $b^{\lambda}$ from the budget constraint in the state the household is employed full-time only to obtain

$$
b^{\lambda}=c^{e, f}+p^{q}\left(c^{u}-\pi\right)-w \bar{h}-\pi .
$$

Next, plug the obtained expression in the budget constraint in the state when the household is employed overtime to obtain

$$
\begin{gathered}
c^{e, o}+p^{q}\left(1+p^{\lambda}\right) c^{u}+p^{\lambda} c^{e, f}+w^{o} h^{o}+\left(1+p^{\lambda}\right) w \bar{h}+ \\
+\left(1+p^{q}+p^{\lambda} p^{q}\right) \pi .
\end{gathered}
$$

The problem now simplifies to

$$
\begin{aligned}
& \max _{\sigma \in \Sigma} q(i)\left\{\lambda(i)\left[\ln \left(c^{e, f}\right)+\alpha \ln (1-\bar{h})\right]+(1-\lambda(i)) *\right. \\
& \left.*\left[\ln \left(c^{e, o}\right)+\alpha \ln \left(1-\bar{h}-h^{o}\right)\right]\right\}+[1-q(i)] \ln \left(c^{u}\right)
\end{aligned}
$$


s.t

$$
\begin{gathered}
c^{e, o}+p^{q}\left(1+p^{\lambda}\right) c^{u}+p^{\lambda} c^{e, f}+w^{o} h^{o}+\left(1+p^{\lambda}\right) w \bar{h}+ \\
+\left(1+p^{q}+p^{\lambda} p^{q}\right) \pi .
\end{gathered}
$$

First-order optimality conditions (where $\mu$ is the Lagrange multiplier attached to the constraint):

$$
\begin{aligned}
c^{e, f} & : \frac{q \lambda}{c^{e, f}}=p^{\lambda} \mu \\
c^{e, o} & : \frac{q(1-\lambda)}{c^{e, o}}=\mu \\
c^{u} & : \frac{1-\lambda}{c^{u}}=\mu p^{q}\left(1+p^{\lambda}\right) .
\end{aligned}
$$

Since we already established that consumption will be equalized across all states, we can obtain (divide optimality conditions for full-time and unemployed)

$$
p^{\lambda}=\frac{\lambda}{1-\lambda}
$$

that is, the price of insurance equals the odds ratio of being chosen to work.

$$
\begin{aligned}
q: \quad & \lambda[\ln (c)+\alpha \ln (1-\bar{h})]+(1-\lambda)\left[\ln (c)+\alpha\left(1-\bar{h}-h^{o}\right)\right]- \\
& -\ln (c)=0 .
\end{aligned}
$$

Hence,

$$
\lambda=\frac{\ln \left(1-\bar{h}-h^{o}\right)}{\ln (1-\bar{h})-\ln \left(1-\bar{h}-h^{o}\right)} \in(0,1) .
$$

With the obtained value for $\lambda$ we can solve for $p^{\lambda}$. Divide throughout the optimality conditions for overtime employed and unemployed to obtain

$$
\frac{q(1-\lambda)}{1-q}=\frac{1}{p^{q}\left(1+p^{\lambda}\right)}
$$


since

$$
1+p^{\lambda}=\frac{1}{1-\lambda}
$$

it follows

$$
\frac{q(1-\lambda)}{1-q}=\frac{1-\lambda}{p^{q}}
$$

or

$$
\frac{q}{1-q}=\frac{1}{p^{q}},
$$

which (the odds ratio being chosen to work) characterizes optimal q.

2. Firm maximization condition. Taking prices $w, w^{o}, \pi$ as given,

$$
\max _{\bar{H}, H^{o}} F\left(\bar{H}, H^{o}\right)-w \bar{H}-w^{o} H^{o} \quad \text { s.t. } \quad \bar{H} \geq 0, H^{o} \geq 0 .
$$

3. Insurance markets: Insurance companies maximize profit.

The insurance is sequential. In stage 1 , by taking $p^{q}(i)$ as given, $b^{q}(i)$ solves

$$
\max _{b^{q}} q p^{q}(i) b^{q}-(1-q) b^{q}
$$

i.e. the revenue if individual is working minus payment is $\mathrm{s} / \mathrm{he}$ is not, or the proportion of people working and contributing towards the unemployment benefits pool and the distribution of benefits to the unemployed.

In the second stage, a separate insurance scheme is run among those that are selected for employment. Taking $p^{\lambda}(i)$ as given, $b^{\lambda}(i)$ solves

$$
\max _{b^{\lambda}} q\left[(1-\lambda) p^{\lambda}(i) b^{\lambda}-\lambda b^{\lambda}\right]
$$


i.e. the revenue if, conditional on being employed, an individual is working overtime $(1-\lambda)$ minus payment is $\mathrm{s} /$ he is not, or the proportion of people working overtime and contributing towards the benefits pool for those who are not selected for extra hours and the distribution of benefits to the full-time employees. In a way, the extra gains (compensate) from the over-time wage premium are shared with the workers who do not do overtime. This implicitly clears the insurance market for each individual.

In equilibrium, the price of insurance depends on the probability of the event the household is insuring against. We cannot enforce $p^{q}(i)=p^{q}$ and $p^{\lambda}(i)=p^{\lambda}$ although ex post (in equilibrium) that would indeed be the case. For the insurance firm, the profits are linear in $p^{q}$ and $p^{\lambda}$. This implies that profits cannot be positive or negative in equilibrium, but have to be zero. Zero profits means that

$$
p^{\lambda}=\frac{\lambda}{1-\lambda} \text { and } p^{q}=\frac{1-q}{q} .
$$

A common interpretation is that for insurance companies the price of the insurance is the odds ratio, or the ratio of probabilities of the two events. $q$ is the same for all households, and $\lambda$ is the same for all employed households.

\section{Market-clearing conditions:}

$$
\begin{array}{r}
\int_{i} q(i) \bar{h} d i=\bar{H}, \\
\int_{i} q(i)[1-\lambda(i)] h^{o} d i=H^{o}, \\
\int_{i}\left\{q(i)\left[\lambda(i) c^{e, f}+(1-\lambda(i)) c^{e, o}\right]+\right. \\
\left.+[1-q(i)] c^{u}\right\} d i=F\left(\bar{H}, H^{o}\right),
\end{array}
$$

The consumers will buy full insurance to equalize consumption in all states (employed, unemployed), (full-time employed-employed), 
(overtime work - employed). In particular, when income is stochastic, i.e., it is uncertain whether the individual will be employed, we need the insurance market to offer conditional insurance. This is because of the sequential non-convexity of the labor choice set, which is similar to having incomplete markets. Lotteries then can be introduced to achieve market completeness. Therefore, randomization may be optimal in a non-convex environment even though there is no aggregate uncertainty. $]^{7}$

\section{Conclusions}

This note describes the lottery- and insurance-market equilibrium in an economy with straight-time and overtime employment. In contrast to Hansen and Sargent (1988), the overtime-decision is a sequential one. This requires two separate insurance market to operate, one for straight-time work, and one for overtime. Each insurance market would pool the risk of the corresponding group of workers. In addition, given that the labor choice for regular and overtime hours is made in succession, the insurance market for overtime needs to open once the insurance market has closed. In equilibrium, conditional on the two-stage labor supply decision, each household would fully insure against the uncertainty in terms of the employment status. This segmentation and sequentiality features of insurance markets operation is a new result in the literature and a direct consequence of the non-convexity of the labor supply decision, and the sequential nature of the overtime labor decision. The plausibility of the result derived in the paper depends crucially on the fact that probabilities $q$ and $\lambda$ are perfectly observable to everyone, and that the contracts written are perfectly enforceable. Also, who has won and who has lost the

\footnotetext{
${ }^{7}$ Note that with those employment lotteries and insurance, every household enjoys the same level of consumption. The introduction of lotteries in the model achieves perfect consumption smoothing, by breaking the link between the labor choice and the affordable consumption. In equilibrium, not everyone will work, and not everyone will work overtime, but everyone will consume the same.
} 
lottery is assumed to be perfect knowledge. Lastly, everyone will always announce truthfully the same $q$ and $\lambda$ to the in- surance companies. Therefore, whether and how this insurance-market sequentiality can be implemented in reality is not entirely clear at this point.

\section{References}

Cooley, T. and E. Prescott (1995). Economic Growth and Business Cycles. In T. Cooley (Ed.), Frontiers of Business Cycle Research. Princeton NJ, Princeton University Press.

Hansen, G. (1985). Indivisible Labor and the Business Cycle. Journal of Monetary Economics 16, 309-328.

Hansen, G. and T. Sargent (1988). Straight time and overtime in Equilibrium. Journal of Monetary Economics 21, 281-308.

Kydland, F. (1995). Business Cycles and Aggregate Labor Market Fluctuations. In T. Cooley (Ed.), Frontiers of Business Cycle Research. Princeton NJ, Princeton University Press.

Rogerson, R. (1988). Indivisible Labor, lotteries and equilibrium. Journal of Monetary Economics 21, 3-16.

Vasilev, A. (2016). Straight-time and Overtime: A Sequential-Lottery Approach. Theoretical and Practical Research in Economic Fields 13(1), 1-5. 